\title{
Synthesis of new trifluoromethyl-containing cycloalka[b]quinolines derived from alkoxycycloalkenes
}

\author{
Helio G. Bonacorso,* Tatiana S. Moraes, Nilo Zanatta, Marcos A. P. Martins, \\ and Alex F. C. Flores
}

Nucleo de Química de Heterociclos (NUQUIMHE), Departamento de Química, Universidade Federal de Santa Maria, 97105-900 - Santa Maria, RS - Brazil

E-mail: heliogb@base.ufsm.br

\begin{abstract}
The synthesis of a series of trifluoromethyl-cycloalka[b]quinolines (5a, 6a, 6c-e) in 15-30\% yields, by intramolecular cyclization reactions of the readily available intermediates 2trifluoroacetyl-1-(arylamino)-cycloalkenes (3a-f and 4a-f) using poly-phosphoric acid (PPA) is reported. Compounds $\mathbf{3}$ and $\mathbf{4}$ are obtained from the reaction of 2-trifluoroacetyl-1methoxycycloalkenes 1 ( $n=1$ and 3 ) with six 4- substituted anilines 2, where the 4- substituents are $\mathrm{Me}, \mathrm{OMe}, \mathrm{NO}_{2}, \mathrm{Cl}, \mathrm{Br}$, I, in acetonitrile under reflux.
\end{abstract}

Keywords: Quinolines, cycloalka[b]quinolines, cycloalkenes, PPA

\section{Introduction}

Malaria is a major parasitic disease affecting over 100 countries of the tropical and subtropical regions of the world. Around 300-500 million clinical cases of malaria are reported every year, of which around 2-3 million die due to complications. Plasmodium falciparum is the parasite responsible for most malarial deaths. Chloroquine remains a major antimalarial drug, but its efficacy and that of other chemotherapeutic agents such as mefloquine is being steadily lessened by the spread of resistant parasites. Thus, the development of alternative drugs is a continuing and urgent necessity. ${ }^{1}$

Quinolines possess important pharmacological properties. ${ }^{2}$ Members of this family have wide applications in medicinal chemistry, being used as antimalarial, anti-inflammatory, antiasthmatic, antibacterial, antihypertensive, and tyrosine-kinase- inhibiting agents. In addition, quinolines are valuable synthons used for the preparation of nano- and meso- structures with enhanced electronic and photonic properties. Despite their importance from pharmacological, industrial, and synthetic points of view, comparatively few methods for their preparation have been reported. ${ }^{3}$ One of the most effective is the Friedländer quinoline synthesis, wherein an 
amino-arene incorporating an ortho- tethered enal or enone moiety engages in an intramolecular Schiff base condensation or related reaction to deliver the target heterocycle. In addition to conventional and well-known methods such as the Skraup, Döbner-von Miller, Conrad Limpach and Pfitzinger syntheses, homogeneous transition metal-catalyzed synthetic methods have been attempted because of the wide availability of substrate. ${ }^{4}$

On the other hand, fluorine substitution can alter the chemical properties, disposition, and biological activity of many drugs. Many fluorinated compounds have been widely used in the treatment of diseases. These include antidepressants, anti-inflammatory agents, antimalarial drugs, antipsychotics, antiviral agents, steroids, and general anaesthetics. Moreover, it has been reported that the introduction of a trifluoromethyl and its higher homologue $\mathrm{C}_{n} \mathrm{~F}_{2 n+1}$ groups into a heterocycle, frequently results in much more potent activity than that of the parent compound, a fact which is probably related to the high lipophylicity of perfluoroalkyl substituents. ${ }^{5,6}$

A review of the literature has shown that the synthesis of trifluoromethylated quinolines and derivatives has been little explored. In 2006, we described the synthesis of a series of 4-[3alkyl(aryl)-5-trifluoromethyl-1H-pyrazol-1-yl]-7-chloroquinolines ${ }^{7}$ from cyclocondensation reactions between 4-methoxy-1,1,1-trifluoro-3-buten-2-ones and 7-chloro-4-hydrazoquinoline, which yielded the 4-[3-alkyl(aryl)-5-hydroxy-5-trifluoromethyl-4,5-dihidro-1H-pyrazol-1-yl]-7chloroquinolines. These 2-pyrazoline intermediates presented enhanced antimalarial activity against the Plasmodium falciparum parasite, ${ }^{7}$ when compared with the most commonly used anti-malarial agents, Chloroquine and Amodiaquine.

Surya and Gibbs have developed a very simple and efficient but very expensive new method to obtain polycyclic quinolines by a Friedländer condensation involving 2-aminoarylketones with cyclic ketones in the presence of catalysis of yttrium triflate. ${ }^{2}$ Other well-known methods to obtain quinolines include using microwave as reported by Asís et al. ${ }^{1}$ or employing ruthenium catalysis as described by Shim and Cho. ${ }^{8}$ Recently, Banwell reported the synthesis of some quinolines by an Ullmann coupling mediated by palladium catalysis. ${ }^{4}$

In 2002, we published a convenient method to obtain a series of 3-alkyl-2-aryl-4trifluoromethylbenzo[h]quinolines by intramolecular cyclization of the readily available intermediates, $\quad N$-(2-alkyl-1-aryl-3-oxo-4,4,4-trifluorobutenyl)-1-naphthylamines, using polyphosphoric acid. ${ }^{9}$

More recently, we have also presented the synthesis of a series of $N$-aryl-2-trifluoroacetyl3,4-dihydro-naphthylamines by the methoxy-arylamino exchange reaction of 1-methoxy-2trifluoroacetyl-3,4-dihydro-naphthalene with various anilines. Subsequently, an intramolecular cyclization reaction of these 3,4-dihydro-naphthylamines carried out in polyphosphoric acid and in the absence of solvent afforded the corresponding series of 7-trifluoromethyl-5,6dihydrobenzo[c]acridines. ${ }^{10}$

The present study presents a general method to regiospecifically synthesize $\beta$-enaminoketones $\mathbf{3}$ and $\mathbf{4}$ and trifluoromethyl-cycloalka[b]quinolines $\mathbf{5}$ and $\mathbf{6}$ from the reaction of cyclic $\beta$-methoxyvinyl trifluoromethyl ketones $1(n=1$ and 3$)$ with six substituted anilines 2 (Scheme 1). Although many methods for the synthesis of quinolines 5 and $\mathbf{6}$ have been published, none of 
them have employed the strategy adopted in this study. Our strategy has the following advantages: (i) It is easier to introduce the substituent at position 7 or 2 of the quinoline ring; (ii) the reactivity of the $\beta$-carbon in $\mathbf{1}$ and the carbonyl carbon in $\mathbf{3}$ and $\mathbf{4}$ allow the regiospecific introduction of a wide scope of anilines bearing both electron- donor and electron- withdrawing substituents.

\section{Results and Discussion}

The $\beta$-methoxyvinyl trifluoromethyl ketones 1 derived from the trifluoroacetylation reaction of cycloalkadimethyl acetals were prepared previously. ${ }^{11-14}$

Firstly, two novel series of twelve 2-trifluoroacetyl-1-(arylamino)-cycloalkenes (3a-f, 4a-f) were obtained in $35-78 \%$ yields by the $O, N$-exchange reaction of 2-trifluoroacetyl-1methoxycycloalkenes, 1, with 4-substituted anilines, 2. These reactions were carried out in acetonitrile under reflux for $24 \mathrm{~h}$, by a methodology similar to that which we described previously. ${ }^{9,10}$ The best results were obtained when a solution of $\mathbf{2}$ in anhydrous acetonitrile was added dropwise to $\mathbf{1}$, also diluted in the same solvent.

Secondly, the intramolecular cyclization reactions of 2-trifluoroacetyl-1-(arylamino)cycloalkenes (3a-f and 4a-f) were carried out in polyphosphoric acid (PPA) in the absence of solvent according to the method described in our earlier work. ${ }^{9,10}$ At first, PPA $\left(\mathrm{P}_{2} \mathrm{O}_{5}+\mathrm{H}_{3} \mathrm{PO}_{4}\right)$ was prepared at $90{ }^{\circ} \mathrm{C}$ and the compounds 3a-f and 4a-f were added in small portions to the acid mixture. The cyclizations of 3a-f and 4a-f only proceeded at $90-110{ }^{\circ} \mathrm{C}$ for $24 \mathrm{~h}$ to afford the corresponding new series of five trifluoromethyl-cycloalka[b]quinolines (5a , 6a, 6c-e) in $15-$ $30 \%$ yields. To search for better reaction conditions and results, we tested some Lewis acids and reactions under microwave conditions. Unfortunately, $\mathrm{POCl}_{3}{ }^{15}, \mathrm{P}_{2} \mathrm{O}_{5} / \mathrm{POCl}_{3}{ }^{16}$ and trifluoroacetic acid $^{17}$ as well as microwave conditions produced no positive results for any of the quinolines, and especially for the methoxy (6b) and iodo (5f and $\mathbf{6 f}$ )- derivatives, which were not isolated.

The unambiguous ${ }^{1} \mathrm{H}$ - and ${ }^{13} \mathrm{C}$ - NMR chemical shift assignments of compounds 1, 3a-f, 4a-f, 5a and 6a, 6c-e were made with the help of HETCORR and COLOQ 2D-NMR experiments and by comparison with NMR data of similar compounds reported previously. ${ }^{9,10}$

\section{Conclusions}

In conclusion, we have developed a convenient procedure to obtain new trifluoromethylcycloalka $[b]$ quinolines as heteropolycycles derived from cycloalkanones and 4-substituted anilines involving the isolation of 2-trifluoroacetyl-1-arylamino-cycloalkene intermediates. Furthermore, we have been able to synthesize and use for the first time 1-methoxy-2trifluoroacetyl-cycloalkene in an enaminone synthesis and in intramolecular cyclocondensation reactions. We hope that the new trifluoromethyl substituted quinolines described here contribute 
to a combinatorial library of quinoline derivatives aiming to determine a more potent drug and its action pathway as an antimalarial agent.<smiles>COC1=C(C(=O)C(F)(F)F)[AlH]CC1</smiles>

1

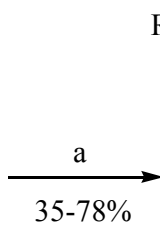<smiles>[R]c1ccc(NC2=C(C(=O)C(F)(F)F)[AlH]CC2)cc1</smiles>

3a-f $(n=1)$ 4a-f $(n=3)$

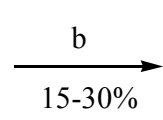<smiles>[R]c1ccc2nc3c(c(C(F)(F)F)c2c1)[AlH]CC3</smiles>

5 a $(n=1)$ 6 a, c, d, e (n=3)

\begin{tabular}{c|cccccc} 
2-6 & $\mathbf{a}$ & $\mathbf{b}$ & $\mathbf{c}$ & $\mathbf{d}$ & $\mathbf{e}$ & $\mathbf{f}$ \\
\hline $\mathrm{R}$ & $\mathrm{Me}$ & $\mathrm{OMe}$ & $\mathrm{NO}_{2}$ & $\mathrm{Cl}$ & $\mathrm{Br}$ & $\mathrm{I}$
\end{tabular}

( a ) $4-\mathrm{NH}_{2} \mathrm{C}_{6} \mathrm{H}_{4} \mathrm{R}$ (2a-f), MeCN, reflux, $24 \mathrm{~h}$.

(b) PPA, $90-120^{\circ} \mathrm{C}, 24 \mathrm{~h}$.

\section{Scheme 1}

\section{Experimental Section}

General Procedures. Unless otherwise indicated all common reagents and solvents were used as obtained from commercial suppliers without further purification. RT denotes room temperature. The melting points were determined on a Reichert Thermovar apparatus and are uncorrected. ${ }^{1} \mathrm{H}$ - and ${ }^{13} \mathrm{C}$ - NMR spectra were acquired on a Bruker DPX $200\left({ }^{1} \mathrm{H}\right.$ - at 200.13 $\mathrm{MHz})$ and Bruker DPX $400\left({ }^{1} \mathrm{H}-\right.$ at $400.13 \mathrm{MHz}$ and ${ }^{13} \mathrm{C}$ at $\left.100.61 \mathrm{MHz}\right)$ spectrometer, $5 \mathrm{~mm}$ sample tubes, $298 \mathrm{~K}$, digital resolution $\pm 0.01 \mathrm{ppm}$, in $\mathrm{CDCl}_{3}$ and using TMS as internal reference. Mass spectra were registered in a HP 6890 GC connected to a HP 5973 MSD and interfaced by a Pentium PC. The GC was equipped with a split-splitless injector, autosampler, cross-linked HP-5 capillary column (30 m, $0.32 \mathrm{~mm}$ of internal diameter), and helium was used as the carrier gas. The CHN elemental analyses were performed on a Perkin-Elmer $2400 \mathrm{CHN}$ elemental analyzer (São Paulo University, USP/Brazil).

General procedure for the synthesis of 2-trifluoroacetyl-1-(arylamino)-cyclopentene (3a-f) and 2-trifluoroacetyl-1-(arylamino)-cycloheptene (4a-f)

To a magnetically stirred solution of $\mathbf{1}(1.04 \mathrm{~g}, 5 \mathrm{mmol})$ in anhydrous acetonitrile $(12 \mathrm{~mL})$ was added dropwise a solution of aniline $2 \mathbf{a}-\mathbf{f}(5 \mathrm{mmol})$ in anhydrous acetonitrile $(5 \mathrm{~mL})$ at RT. The 
mixture was stirred under reflux for $24 \mathrm{~h}$. The solvent was evaporated under reduced pressure and the solid products 3a-f and 4a-f were recrystallized from ethanol (yields 35-78\%).

2-Trifluoroacetyl-1-(4-methylphenylamino)-cyclopentene (3a). Yellow solid, yield 40\%, Mp. 68-70 ${ }^{\circ} \mathrm{C} .{ }^{1} \mathrm{H}-\mathrm{NMR}\left(\mathrm{CDCl}_{3}\right): \delta=11.84$ (bs, $\left.1 \mathrm{H}, \mathrm{NH}\right) ; 7.17(\mathrm{~d}, 2 \mathrm{H}, J=8.2, \mathrm{Ar}) ; 7.03(\mathrm{~d}, 2 \mathrm{H}, J=$ 8.5, Ar); 2.81-2.73 (m, 4H, $\left.\mathrm{CH}_{2}\right) ; 2.34$ (s, 3H, Me); 2.00-1.86 (m, 2H, $\left.\mathrm{CH}_{2}\right) .{ }^{13} \mathrm{C}-\mathrm{NMR}\left(\mathrm{CDCl}_{3}\right)$ : $\delta=173.9\left(\mathrm{q},{ }^{2} J_{\mathrm{C}-\mathrm{F}}=33.6, \mathrm{C}=\mathrm{O}\right) ; 172.6(\mathrm{C} 1) ; 136.0 ; 135.7 ; 129.9 ; 122.6(6 \mathrm{C}, \mathrm{Ar}) ; 117.7\left(\mathrm{q},{ }^{1} J_{\mathrm{C}-\mathrm{F}}\right.$ $\left.=288.4, \mathrm{CF}_{3}\right) ; 103.1(\mathrm{C} 2) ; 33.1\left(\mathrm{CH}_{2}\right) ; 27.4\left(\mathrm{q},{ }^{4} J_{\mathrm{C}-\mathrm{F}}=2.8, \mathrm{C} 3\right) ; 22.8\left(\mathrm{CH}_{2}\right) ; 20.8(\mathrm{Me})$. CG-MS (EI, $70 \mathrm{eV}): m / z(\%)=269\left(\mathrm{M}^{+}, 43\right) ; 200(100) ; 172(9) ; 91$ (17). Anal. Calcd.. for $\mathrm{C}_{14} \mathrm{H}_{14} \mathrm{~F}_{3} \mathrm{NO}$ (269.1): C, 62.45; H, 5.24; N, 5.20. Found: C, 62.13; H, 5.16; N, 5.15\%.

2-Trifluoroacetyl-1-(4-methoxyphenylamino)-cyclopentene (3b). Yellow solid, yield 46\%, Mp. 63-64 ${ }^{\circ} \mathrm{C} .{ }^{1} \mathrm{H}-\mathrm{NMR}\left(\mathrm{CDCl}_{3}\right): \delta=11.75$ (bs, $\left.1 \mathrm{H}, \mathrm{NH}\right) ; 7.08$ (d, 2H, J=9.0, Ar); 6.90 (d, 2H, $J=8.7$, Ar); $3.81(\mathrm{~s}, 3 \mathrm{H}, \mathrm{OMe}) ; 2.78-2.67\left(\mathrm{~m}, 4 \mathrm{H}, \mathrm{CH}_{2}\right) ; 1.96-1.89$ (m, 2H, $\left.\mathrm{CH}_{2}\right) .{ }^{13} \mathrm{C}-\mathrm{NMR}$ $\left(\mathrm{CDCl}_{3}\right): \delta=173.3\left(\mathrm{q},{ }^{2} J_{\mathrm{C}-\mathrm{F}}=33.7, \mathrm{C}=\mathrm{O}\right) ; 173.1(\mathrm{C} 1) ; 157.9 ; 131.2 ; 124.4 ; 114.4(6 \mathrm{C}, \mathrm{Ar})$; $117.7\left(\mathrm{q},{ }^{1} J_{\mathrm{C}-\mathrm{F}}=288.9, \mathrm{CF}_{3}\right) ; 102.7(\mathrm{C} 2) ; 55.3(\mathrm{OMe}) ; 32.9\left(\mathrm{CH}_{2}\right) ; 27.5\left(\mathrm{q},{ }^{4} J_{\mathrm{C}-\mathrm{F}}=2.8, \mathrm{C} 3\right) ; 22.7$ $\left(\mathrm{CH}_{2}\right)$. CG-MS (EI, $\left.70 \mathrm{eV}\right): \mathrm{m} / \mathrm{z}(\%)=285\left(\mathrm{M}^{+}, 64\right) ; 216$ (100); 187 (7); 158 (3); 173 (51); 77(8). Anal. Calcd. for $\mathrm{C}_{14} \mathrm{H}_{14} \mathrm{~F}_{3} \mathrm{NO}_{2}$ (285.1): C, 58.95; H, 4.95; N, 4.91. Found: C, 59.23; H, 4.64; N, 4.93\%.

2-Trifluoroacetyl-1-(4-nitrophenylamino)-cyclopentene (3c). Black solid, yield 63\%, Mp. 112-114 ${ }^{\circ} \mathrm{C} .{ }^{1} \mathrm{H}-\mathrm{NMR}\left(\mathrm{CDCl}_{3}\right): \delta=11.99$ (bs, $\left.1 \mathrm{H}, \mathrm{NH}\right) ; 8.25(\mathrm{~d}, 2 \mathrm{H}, J=9.2, \mathrm{Ar}) ; 7.26(\mathrm{~d}, 2 \mathrm{H}, J$ $=9.0$, Ar); 3.03-2.79 (m, 4H, $\left.\mathrm{CH}_{2}\right) ; 2.17-1.99\left(\mathrm{~m}, 2 \mathrm{H}, \mathrm{CH}_{2}\right) .{ }^{13} \mathrm{C}-\mathrm{NMR}\left(\mathrm{CDCl}_{3}\right): \delta=176.3(\mathrm{q}$, $\left.{ }^{2} J_{\mathrm{C}-\mathrm{F}}=34.5, \mathrm{C}=\mathrm{O}\right) ; 169.4(\mathrm{C} 1) ; 144.3 ; 144.1 ; 125.4 ; 120.9(6 \mathrm{C}, \mathrm{Ar}) ; 117.1\left(\mathrm{q},{ }^{1} J_{\mathrm{C}-\mathrm{F}}=288.2\right.$, $\left.\mathrm{CF}_{3}\right) ; 106.4(\mathrm{C} 2) ; 33.9\left(\mathrm{CH}_{2}\right) ; 27.4\left(\mathrm{q},{ }^{4} J_{\mathrm{C}-\mathrm{F}}=2.8, \mathrm{C} 3\right) ; 23.0\left(\mathrm{CH}_{2}\right) . \mathrm{CG}-\mathrm{MS}(\mathrm{EI}, 70 \mathrm{eV}): \mathrm{m} / \mathrm{z}(\%)$ $=300\left(\mathrm{M}^{+}, 88\right) ; 231(86) ; 203$ (9); 185 (100); 156 (23); 76 (13). Anal. Calcd. for $\mathrm{C}_{13} \mathrm{H}_{11} \mathrm{~F}_{3} \mathrm{~N}_{2} \mathrm{O}_{3}$ (300.07): C, 52.01; H, 3.69; N, 9.33. Found: C, 52.05; H, 3.68; N, 9.22\%.

1-(4-Chlorophenylamino)-2-trifluoroacetyl-cyclopentene (3d). Yellow solid, yield 73\%, Mp. 76-77 ${ }^{\circ} \mathrm{C} .{ }^{1} \mathrm{H}-\mathrm{NMR}\left(\mathrm{CDCl}_{3}\right): \delta=11.79$ (bs, $\left.1 \mathrm{H}, \mathrm{NH}\right) ; 7.34$ (d, 2H, $\left.J=8.7, \mathrm{Ar}\right) ; 7.08(\mathrm{~d}, 2 \mathrm{H}, J=$ 8.7, Ar); 2.82-2.75 (m, 4H, $\left.\mathrm{CH}_{2}\right) ; 2.03-1.89\left(\mathrm{~m}, 2 \mathrm{H}, \mathrm{CH}_{2}\right) .{ }^{13} \mathrm{C}-\mathrm{NMR}\left(\mathrm{CDCl}_{3}\right): \delta=174.7\left(\mathrm{q},{ }^{2} J_{\mathrm{C}-}\right.$ $\mathrm{F}=33.9, \mathrm{C}=\mathrm{O}) ; 171.7(\mathrm{C} 1) ; 137.0 ; 131.5 ; 129.5 ; 123.8(6 \mathrm{C}, \mathrm{Ar}) ; 117.5\left(\mathrm{q},{ }^{1} J_{\mathrm{C}-\mathrm{F}}=288.6, \mathrm{CF}_{3}\right)$; $103.9(\mathrm{C} 2) ; 33.2\left(\mathrm{CH}_{2}\right) ; 27.5\left(\mathrm{q},{ }^{4} J_{\mathrm{C}-\mathrm{F}}=2.4, \mathrm{C}_{3}\right) ; 22.8\left(\mathrm{CH}_{2}\right)$. CG-MS (EI, $\left.70 \mathrm{eV}\right): \mathrm{m} / \mathrm{z}(\%)=289$ $\left(\mathrm{M}^{+}, 58\right) ; 220$ (100); 184 (13); 156 (8); 111 (11); 75 (9). Anal. Calcd. for $\mathrm{C}_{13} \mathrm{H}_{11} \mathrm{ClF}_{3} \mathrm{NO}$ (289.05): C, 53.90; H, 3.83; N, 4.84. Found: C, 54.0; H, 3.90; N, 4.76\%.

1-(4-Bromophenylamino)-2-trifluoroacetyl-cyclopentene (3e). Yellow solid, yield 52\%, Mp. 68-70 ${ }^{\circ} \mathrm{C} .{ }^{1} \mathrm{H}-\mathrm{NMR}\left(\mathrm{CDCl}_{3}\right): \delta=11.79$ (bs, $\left.1 \mathrm{H}, \mathrm{NH}\right) ; 7.49$ (d, 2H, $\left.J=9.0, \mathrm{Ar}\right) ; 7.03$ (d, 2H, $J=$ 8.7, Ar); 2.83-2.75 (m, 4H, $\left.\mathrm{CH}_{2}\right) ; 2.04-1.89\left(\mathrm{~m}, 2 \mathrm{H}, \mathrm{CH}_{2}\right) .{ }^{13} \mathrm{C}-\mathrm{NMR}\left(\mathrm{CDCl}_{3}\right): \delta=174.8\left(\mathrm{q},{ }^{2} J_{\mathrm{C}-}\right.$ $\mathrm{F}=34.1, \mathrm{C}=\mathrm{O}) ; 171.6(\mathrm{C} 1) ; 137.5 ; 132.4 ; 124.0 ; 119.1(6 \mathrm{C}, \mathrm{Ar}) ; 117.5\left(\mathrm{q},{ }^{1} J_{\mathrm{C}-\mathrm{F}}=288.6, \mathrm{CF}_{3}\right)$; $104.0(\mathrm{C} 2) ; 33.2\left(\mathrm{CH}_{2}\right) ; 27.4\left(\mathrm{q},{ }^{4} J_{\mathrm{C}-\mathrm{F}}=2.5, \mathrm{C} 3\right) ; 22.8\left(\mathrm{CH}_{2}\right)$. CG-MS (EI, $\left.70 \mathrm{eV}\right): \mathrm{m} / \mathrm{z}(\%)=333$ $\left(\mathrm{M}^{+}, 74\right) ; 264$ (86); 236 (9); 184 (100); 156 (25); 76 (15). Anal. Calcd. for $\mathrm{C}_{13} \mathrm{H}_{11} \mathrm{BrF}_{3} \mathrm{NO}$ (333.0): C, 46.73; H, 3.32; N, 4.19. Found: C, 46.60; H, 3.08; N, 4.18\%.

2-Trifluoroacetyl-1-(4-iodophenylamino)-cyclopentene (3f). Brown solid, yield 57\%, Mp. 82$84{ }^{\circ} \mathrm{C} .{ }^{1} \mathrm{H}-\mathrm{NMR}\left(\mathrm{CDCl}_{3}\right): \delta=11.79(\mathrm{bs}, 1 \mathrm{H}, \mathrm{NH}) ; 7.68$ (d, 2H, $J=8.7$, Ar); 6.9 (d, 2H, $J=8.7$, 
Ar); 2.84-2.76 (m, 4H, CH$) ; 2.04-1.89\left(\mathrm{~m}, 2 \mathrm{H}, \mathrm{CH}_{2}\right) .{ }^{13} \mathrm{C}-\mathrm{NMR}\left(\mathrm{CDCl}_{3}\right): \delta=174.6\left(\mathrm{q},{ }^{2} J_{\mathrm{C}-\mathrm{F}}=\right.$ $33.9, \mathrm{C}=\mathrm{O}) ; 171.4(\mathrm{C} 1) ; 138.3 ; 138.1 ; 124.0 ; 89.8(6 \mathrm{C}, \mathrm{Ar}) ; 117.5$ (q, $\left.{ }^{1} J_{\mathrm{C}-\mathrm{F}}=288.5, \mathrm{CF}_{3}\right) ; 104.1$ (C2); $33.2\left(\mathrm{CH}_{2}\right) ; 27.4(\mathrm{C} 3) ; 22.8\left(\mathrm{CH}_{2}\right)$. CG-MS (EI, $\left.70 \mathrm{eV}\right): \mathrm{m} / \mathrm{z}(\%)=381\left(\mathrm{M}^{+}, 69\right) ; 312(26)$; 284 (8); 184 (100); 156 (27); 76 (30). Anal. Calcd. for $\mathrm{C}_{13} \mathrm{H}_{11} \mathrm{~F}_{3} \mathrm{INO}$ (380.98): C, 40.97; H, 2.91; N, 3.68. Found: C, 40.81; H, 2.91; N, 3.87\%.

2-Trifluoroacetyl-1-(4-methylphenylamino)-cycloheptene (4a). Yellow solid, yield 53\%, Mp. 90-92 ${ }^{\circ} \mathrm{C} .{ }^{1} \mathrm{H}-\mathrm{NMR}\left(\mathrm{CDCl}_{3}\right): \delta=13.79(\mathrm{bs}, 1 \mathrm{H}, \mathrm{NH}) ; 7.19(\mathrm{~d}, 2 \mathrm{H}, J=7.9, \mathrm{Ar}) ; 6.98(\mathrm{~d}, 2 \mathrm{H}, J=$ 8.2, Ar); 2.61-2.56 (m, 4H, $\left.\mathrm{CH}_{2}\right) ; 2.36(\mathrm{~s}, 3 \mathrm{H}, \mathrm{Me}) ; 1.76-1.62\left(\mathrm{~m}, 6 \mathrm{H}, \mathrm{CH}_{2}\right) .{ }^{13} \mathrm{C}-\mathrm{NMR}\left(\mathrm{CDCl}_{3}\right)$ : $\delta=174.9(\mathrm{C} 1) ; 173.9\left(\mathrm{q},{ }^{2} J_{\mathrm{C}-\mathrm{F}}=31.0, \mathrm{C}=\mathrm{O}\right) ; 136.9 ; 134.7 ; 129.8 ; 125.3(6 \mathrm{C}, \mathrm{Ar}) ; 118.6\left(\mathrm{q},{ }^{1} J_{\mathrm{C}-\mathrm{F}}\right.$ $\left.=288.7, \mathrm{CF}_{3}\right) ; 103.9(\mathrm{C} 2) ; 30.7\left(\mathrm{CH}_{2}\right) ; 29.9\left(\mathrm{CH}_{2}\right) ; 28.2\left(\mathrm{CH}_{2}\right) ; 25.6\left(\mathrm{CH}_{2}\right) ; 25.4\left(\mathrm{q},{ }^{4} J_{\mathrm{C}-\mathrm{F}}=3.1\right.$, C3); 20.7 (Me). CG-MS (EI, 70 eV): m/z (\%) = $297\left(\mathrm{M}^{+}, 55\right) ; 228$ (100); 214 (2); 200 (33); 91 (45). Anal. Calcd. for $\mathrm{C}_{16} \mathrm{H}_{18} \mathrm{~F}_{3} \mathrm{NO}$ (297.13): C, 64.64; H, 6.10; N, 4.71. Found: C, 64.43; H, 5.92; N, 4.79\%.

2-Trifluoroacetyl-1-(4-methoxyphenylamino)-cycloheptene (4b). Yellow solid, yield 35\%, Mp. 70-72 ${ }^{\circ} \mathrm{C} .{ }^{1} \mathrm{H}-\mathrm{NMR}\left(\mathrm{CDCl}_{3}\right): \delta=13.72(\mathrm{bs}, 1 \mathrm{H}, \mathrm{NH}) ; 7.02$ (d, 2H, J=9.2, Ar); 6.89 (d, 2H, $J=9.0$, Ar); 3.81 (s, 3H, OMe); 2.59-2.54 (m, 4H, $\left.\mathrm{CH}_{2}\right) ; 1.74-1.61\left(\mathrm{~m}, 6 \mathrm{H}, \mathrm{CH}_{2}\right) .{ }^{13} \mathrm{C}-\mathrm{NMR}$ $\left(\mathrm{CDCl}_{3}\right): \delta=175.1(\mathrm{C} 1) ; 173.9$ (q, $\left.{ }^{2} J_{\mathrm{C}-\mathrm{F}}=30.9, \mathrm{C}=\mathrm{O}\right) ; 158.5 ; 130.2 ; 126.8 ; 114.4(6 \mathrm{C}, \mathrm{Ar})$; $118.7\left(\mathrm{q},{ }^{1} J_{\mathrm{C}-\mathrm{F}}=288.9, \mathrm{CF}_{3}\right) ; 103.8(\mathrm{C} 2) ; 55.3(\mathrm{OMe}) ; 30.7\left(\mathrm{CH}_{2}\right) ; 29.9\left(\mathrm{CH}_{2}\right) ; 28.3\left(\mathrm{CH}_{2}\right) ; 25.6$ $\left(\mathrm{CH}_{2}\right) ; 25.4$ (q, $\left.{ }^{4} J_{\mathrm{C}-\mathrm{F}}=3.0, \mathrm{C} 3\right)$. CG-MS (EI, $\left.70 \mathrm{eV}\right): \mathrm{m} / \mathrm{z}(\%)=313\left(\mathrm{M}^{+}, 99\right) ; 244(100) ; 216$ (50); 122 (49); 108 (17). Anal. Calcd. for $\mathrm{C}_{16} \mathrm{H}_{18} \mathrm{~F}_{3} \mathrm{NO}_{2}$ (313.13): C, 61.33; H, 5.79; N, 4.47. Found: C, 61.05; H, 5.66; N, 4.37\%.

2-Trifluoroacetyl-1-(4-nitrophenylamino)-cycloheptene (4c). Yellow solid, yield 78\%, Mp. 126-128 ${ }^{\circ} \mathrm{C} .{ }^{1} \mathrm{H}-\mathrm{NMR}\left(\mathrm{CDCl}_{3}\right): \delta=13.74(\mathrm{bs}, 1 \mathrm{H}, \mathrm{NH}) ; 8.27(\mathrm{~d}, 2 \mathrm{H}, J=9.0, \mathrm{Ar}) ; 7.22(\mathrm{~d}, 2 \mathrm{H}, J$ $=9.0, \mathrm{Ar}) ; 2.72-2.57\left(\mathrm{~m}, 4 \mathrm{H}, \mathrm{CH}_{2}\right) ; 1.83-1.63\left(\mathrm{~m}, 6 \mathrm{H}, \mathrm{CH}_{2}\right) .{ }^{13} \mathrm{C}-\mathrm{NMR}\left(\mathrm{CDCl}_{3}\right): \delta=176.3(\mathrm{q}$,

$\left.{ }^{2} J_{\mathrm{C}-\mathrm{F}}=32.5, \mathrm{C}=\mathrm{O}\right), 172.4(\mathrm{C} 1), 145.4 ; 143.5 ; 125.0 ; 124.9(6 \mathrm{C}, \mathrm{Ar}) ; 117.9\left(\mathrm{q},{ }^{1} J_{\mathrm{C}-\mathrm{F}}=288.0\right.$, $\left.\mathrm{CF}_{3}\right) ; 106.7(\mathrm{C} 2) ; 30.7\left(\mathrm{CH}_{2}\right) ; 30.1\left(\mathrm{CH}_{2}\right) ; 27.6\left(\mathrm{CH}_{2}\right) ; 25.5\left(\mathrm{CH}_{2}\right) ; 25.4(\mathrm{C} 3)$. CG-MS (EI, 70 $\mathrm{eV}): m / z(\%)=328\left(\mathrm{M}^{+}, 100\right) ; 259(88) ; 231$ (37); 213 (64); 76 (25). Anal. Calcd. for $\mathrm{C}_{15} \mathrm{H}_{15} \mathrm{~F}_{3} \mathrm{~N}_{2} \mathrm{O}_{3}$ (328.1): C, 54.88; H, 4.61; N, 8.53. Found: C, 54.76; H, 4.51; N, 8.58\%.

1-(4-Clorophenylamino)-2-trifluoroacetyl-cycloheptene (4d). Brown solid, yield 76\%, Mp. 93-94 ${ }^{\circ} \mathrm{C} .{ }^{1} \mathrm{H}-\mathrm{NMR}\left(\mathrm{CDCl}_{3}\right): \delta=13.75$ (bs, $\left.1 \mathrm{H}, \mathrm{NH}\right) ; 7.37$ (d, 2H, J=8.7, Ar); 7.03 (d, 2H, $J=$ 8.5, Ar); 2.61-2.56 (m, 4H, $\left.\mathrm{CH}_{2}\right) ; 1.77-1.62\left(\mathrm{~m}, 6 \mathrm{H}, \mathrm{CH}_{2}\right) .{ }^{13} \mathrm{C}-\mathrm{NMR}\left(\mathrm{CDCl}_{3}\right): \delta=174.8\left(\mathrm{q},{ }^{2} J_{\mathrm{C}-}\right.$ $\mathrm{F}=31.2, \mathrm{C}=\mathrm{O}) ; 174.1(\mathrm{C} 1) ; 136.1 ; 132.7 ; 129.5 ; 126.8(6 \mathrm{C}, \mathrm{Ar}) ; 118.5\left(\mathrm{q},{ }^{1} J_{\mathrm{C}-\mathrm{F}}=288.9, \mathrm{CF}_{3}\right)$; $104.7(\mathrm{C} 2) ; 30.7\left(\mathrm{CH}_{2}\right) ; 30.0\left(\mathrm{CH}_{2}\right) ; 28.1\left(\mathrm{CH}_{2}\right) ; 25.6\left(\mathrm{CH}_{2}\right) ; 25.4\left(\mathrm{q},{ }^{4} J_{\mathrm{C}-\mathrm{F}}=3.1, \mathrm{C} 3\right)$. CG-MS $(\mathrm{EI}, 70 \mathrm{eV}): \mathrm{m} / \mathrm{z}(\%)=317\left(\mathrm{M}^{+}, 71\right) ; 248$ (100); 220 (28); 111 (25); 75 (17). Anal. Calcd. for $\mathrm{C}_{15} \mathrm{H}_{15} \mathrm{ClF}_{3} \mathrm{NO}$ (317.08): C, 56.70; H, 4.76; N, 4.41. Found: C, 56.40; H, 4.49; N, 4.22\%.

1-(4-Bromophenylamino)-2-trifluoroacetyl-cycloheptene (4e). Yellow solid, yield 61\%, Mp. 96-98 ${ }^{\circ} \mathrm{C} .{ }^{1} \mathrm{H}-\mathrm{NMR}\left(\mathrm{CDCl}_{3}\right): \delta=13.71(\mathrm{bs}, 1 \mathrm{H}, \mathrm{NH}) ; 7.51$ (d, 2H, $\left.J=8.7, \mathrm{Ar}\right) ; 6.95(\mathrm{~d}, 2 \mathrm{H}, J=$ 8.5, Ar); $2.60-2.54\left(\mathrm{~m}, 4 \mathrm{H}, \mathrm{CH}_{2}\right) ; 1.76-1.59\left(\mathrm{~m}, 6 \mathrm{H}, \mathrm{CH}_{2}\right) .{ }^{13} \mathrm{C}-\mathrm{NMR}\left(\mathrm{CDCl}_{3}\right): \delta=174.9\left(\mathrm{q},{ }^{2} J_{\mathrm{C}-}\right.$ $\mathrm{F}=31.3, \mathrm{C}=\mathrm{O}) ; 173.9(\mathrm{C} 1) ; 136.5 ; 132.4 ; 127.1 ; 120.5(6 \mathrm{C}, \mathrm{Ar}) ; 118.4\left(\mathrm{q},{ }^{1} J_{\mathrm{C}-\mathrm{F}}=288.9, \mathrm{CF}_{3}\right)$; $104.7(\mathrm{C} 2) ; 30.7\left(\mathrm{CH}_{2}\right) ; 30.0\left(\mathrm{CH}_{2}\right) ; 28.0\left(\mathrm{CH}_{2}\right) ; 25.6\left(\mathrm{CH}_{2}\right) ; 25.4\left(\mathrm{q},{ }^{4} J_{\mathrm{C}-\mathrm{F}}=3.2, \mathrm{C} 3\right)$. CG-MS 
(EI, $70 \mathrm{eV}): \mathrm{m} / \mathrm{z}(\%)=361\left(\mathrm{M}^{+}, 77\right) ; 292$ (69); 264 (21); 213 (42); 184 (100); 76 (32). Anal. Calcd. for $\mathrm{C}_{15} \mathrm{H}_{15} \mathrm{BrF}_{3} \mathrm{NO}$ (361.03): C, 49.74; H, 4.17; N, 3.87. Found: C, 49.76; H, 4.12; N, $3.92 \%$

2-Trifluoroacetyl-1-(4-iodophenylamino)-cycloheptene (4f). Yellow solid, yield 61\%, Mp. $112-114{ }^{\circ} \mathrm{C} .{ }^{1} \mathrm{H}-\mathrm{NMR}\left(\mathrm{CDCl}_{3}\right): \delta=13.72(\mathrm{bs}, 1 \mathrm{H}, \mathrm{NH}) ; 7.72(\mathrm{~d}, 2 \mathrm{H}, J=8.2, \mathrm{Ar}) ; 6.84(\mathrm{~d}, 2 \mathrm{H}, J$ $=8.5, \mathrm{Ar}) ; 2.60-2.58\left(\mathrm{~m}, 4 \mathrm{H}, \mathrm{CH}_{2}\right) ; 1.77-1.62\left(\mathrm{~m}, 6 \mathrm{H}, \mathrm{CH}_{2}\right) .{ }^{13} \mathrm{C}-\mathrm{NMR}\left(\mathrm{CDCl}_{3}\right): \delta=174.9(\mathrm{q}$, $\left.{ }^{2} J_{\mathrm{C}-\mathrm{F}}=31.4, \mathrm{C}=\mathrm{O}\right) ; 173.9(\mathrm{C} 1) ; 138.4 ; 137.3 ; 127.4 ; 91.6(6 \mathrm{C}, \mathrm{Ar}) ; 118.4\left(\mathrm{q},{ }^{1} J_{\mathrm{C}-\mathrm{F}}=288.7, \mathrm{CF}_{3}\right)$; $104.8(\mathrm{C} 2) ; 30.8\left(\mathrm{CH}_{2}\right) ; 30.0\left(\mathrm{CH}_{2}\right) ; 28.1\left(\mathrm{CH}_{2}\right) ; 25.6\left(\mathrm{CH}_{2}\right) ; 25.4\left(\mathrm{q},{ }^{4} J_{\mathrm{C}-\mathrm{F}}=3.0, \mathrm{C} 3\right) . \mathrm{CG}-\mathrm{MS}$ $(\mathrm{EI}, 70 \mathrm{eV}): \mathrm{m} / \mathrm{z}(\%)=409\left(\mathrm{M}^{+}, 100\right) ; 340$ (32); 312 (16); 213 (33); 184 (79); 76 (41). Anal. Calcd. for $\mathrm{C}_{15} \mathrm{H}_{15} \mathrm{~F}_{3} \mathrm{INO}$ (409.02): C, 44.03; H, 3.69; N, 3.42. Found: C, 43.69; H, 3.33; N, $3.05 \%$.

General Procedure for the synthesis of trifluoromethyl-cycloalka[b]quinolines (5a, 6a, 6c-e) To a magnetically stirred solution of $\mathrm{H}_{3} \mathrm{PO}_{4}(2 \mathrm{~mL})$ and $\mathrm{P}_{2} \mathrm{O}_{5}(3.0 \mathrm{~g})(\mathrm{PPA})$ at $90{ }^{\circ} \mathrm{C}$, 3a-f and 4a-f ( $5 \mathrm{mmol})$ were added at RT. The mixture was stirred for $24 \mathrm{~h}$ at $110^{\circ} \mathrm{C}$. Distilled water $(10$ $\mathrm{mL})$ and chloroform $(10 \mathrm{~mL})$ were added to the mixture at RT. The solution was then extracted with chloroform (three times, $10 \mathrm{~mL}$ ). The combined organic layers were washed with aq. 10\% solution of $\mathrm{NaOH}$ (three times, $10 \mathrm{~mL}$ ), distilled water (three times, $10 \mathrm{~mL}$ ), dried over anhydrous sodium sulfate and the solvent was removed under reduced pressure. The resulting solid products were recrystallized from ethanol (yields 15-30\%).

9-Trifluoromethyl-7-methyl-2,3-dihydro-1H-cyclopenta[b]quinoline (5a). Black solid, yield 25\%, Mp. 77-78 ${ }^{\circ} \mathrm{C} .{ }^{1} \mathrm{H}-\mathrm{NMR}\left(\mathrm{CDCl}_{3}\right): \delta=7.95(\mathrm{~d}, 1 \mathrm{H}, J=8.5, \mathrm{Ar}) ; 7.84$ (s, 1H, Ar); $7.51(\mathrm{dd}$, $1 \mathrm{H}, J=1.5, J=1.5, \mathrm{Ar}) ; 3.33-3.28\left(\mathrm{~m}, 2 \mathrm{H}, \mathrm{CH}_{2}\right) ; 3.18-3.14\left(\mathrm{~m}, 2 \mathrm{H}, \mathrm{CH}_{2}\right) ; 2.54$ (s, 3H, Me); 2.22-2.16 (m, 2H, $\left.\mathrm{CH}_{2}\right) .{ }^{13} \mathrm{C}-\mathrm{NMR}\left(\mathrm{CDCl}_{3}\right): \delta=167.1(\mathrm{C} 3 \mathrm{a}) ; 146.6(\mathrm{C} 4 \mathrm{a}) ; 136.9(\mathrm{C} 7) ; 133.8(\mathrm{q}$, $\left.{ }^{4} J_{\mathrm{C}-\mathrm{F}}=2.5, \mathrm{C} 8\right) ; 131.1(\mathrm{C} 6) ; 129.0(\mathrm{C} 5) ; 128.1\left(\mathrm{q},{ }^{2} J_{\mathrm{C}-\mathrm{F}}=31.08, \mathrm{C} 9\right) ; 124.6\left(\mathrm{q},{ }^{1} J_{\mathrm{C}-\mathrm{F}}=276.4\right.$, $\left.\mathrm{CF}_{3}\right) ; 122.9\left(\mathrm{q},{ }^{3} J_{\mathrm{C}-\mathrm{F}}=3.0, \mathrm{C} 8 \mathrm{a}\right) ; 122.1(\mathrm{C} 9 \mathrm{a}) ; 34.1\left(\mathrm{CH}_{2}\right) ; 31.1\left(\mathrm{q},{ }^{4} J_{\mathrm{C}-\mathrm{F}}=3.0, \mathrm{C} 1\right) ; 22.8\left(\mathrm{CH}_{2}\right)$; 21.9 (Me). CG-MS (EI, 70 eV): m/z (\%)=251 (M+, 100); 235 (3); 182 (60); 167 (19); 90 (5); 77 (5); 69 (6). Anal. Calcd. for $\mathrm{C}_{14} \mathrm{H}_{12} \mathrm{~F}_{3} \mathrm{~N}$ (251.09): C, 66.93; H, 4.81; N, 5.57. Found: C, 66.85; H, $4.76 ; \mathrm{N}, 5.47 \%$.

11-Trifluoromethyl-2-methyl-7,8,9,10-tetrahydro-6H-cyclohepta[b]quinoline (6a). White solid, yield 30\%, Mp. 92-94 ${ }^{\circ} \mathrm{C} .{ }^{1} \mathrm{H}-\mathrm{NMR}\left(\mathrm{CDCl}_{3}\right): \delta=7.95-7.91(\mathrm{~m}, 2 \mathrm{H}, \mathrm{Ar}) ; 7.50(\mathrm{~d}, 1 \mathrm{H}, J=$ 8.5, Ar); 3.30-3.28 (m, 2H, $\left.\mathrm{CH}_{2}\right) ; 3.11-3.10\left(\mathrm{~m}, 2 \mathrm{H}, \mathrm{CH}_{2}\right) ; 2.54(\mathrm{~s}, 1 \mathrm{H}, \mathrm{Me}) ; 1.90-1.81(\mathrm{~m}, 6 \mathrm{H}$, $\left.\mathrm{CH}_{2}\right) .{ }^{13} \mathrm{C}-\mathrm{NMR}\left(\mathrm{CDCl}_{3}\right): \delta=164.1(\mathrm{C} 5 \mathrm{a}) ; 144.9(\mathrm{C} 4 \mathrm{a}) ; 136.9(\mathrm{C} 2) ; 136.0(\mathrm{C} 10 \mathrm{a}) ; 130.8(\mathrm{C} 3)$; $129.7\left(\mathrm{q},{ }^{2} J_{\mathrm{C}-\mathrm{F}}=30.6, \mathrm{C} 11\right) ; 129.1(\mathrm{C} 4) ; 125.1\left(\mathrm{q},{ }^{1} J_{\mathrm{C}-\mathrm{F}}=278.3, \mathrm{CF}_{3}\right) ; 122.9\left(\mathrm{q},{ }^{4} J_{\mathrm{C}-\mathrm{F}}=5.5, \mathrm{C} 1\right)$; 122.7 (C11a); $39.2\left(\mathrm{CH}_{2}\right) ; 30.9\left(\mathrm{CH}_{2}\right) ; 29.9$ (q, $\left.{ }^{4} \mathrm{~J}_{\mathrm{C}-\mathrm{F}}=4.0, \mathrm{C} 10\right) ; 27.5\left(\mathrm{CH}_{2}\right) ; 26.7\left(\mathrm{CH}_{2}\right) ; 22.1$ (Me). CG-MS (EI, $70 \mathrm{eV}):$ m/z (\%) = $279\left(\mathrm{M}^{+}, 100\right) ; 264$ (65); 210 (34); 195 (11); 77 (5); 69 (2). Anal. Calcd. for $\mathrm{C}_{16} \mathrm{H}_{16} \mathrm{~F}_{3} \mathrm{~N}$ (279.12): C, 68.80; H, 5.77; N, 5.01. Found: C, 68.66; H, 5.63; N, $4.89 \%$.

11-Trifluoromethyl-2-nitro-7,8,9,10-tetrahydro-6H-cyclohepta[b]quinoline (6c). Red solid, yield 15\%, Mp. $141-143{ }^{\circ} \mathrm{C} .{ }^{1} \mathrm{H}-\mathrm{NMR}\left(\mathrm{CDCl}_{3}\right): \delta=9.12(\mathrm{~d}, 1 \mathrm{H}, J=2.0, \mathrm{Ar}) ; 8.47(\mathrm{dd}, 1 \mathrm{H}, J$ 
$=2.0, \mathrm{~J}=2.0, \mathrm{Ar}) ; 8.30(\mathrm{~d}, 1 \mathrm{H}, J=9.2, \mathrm{Ar}) ; 3.46-3.41\left(\mathrm{~m}, 2 \mathrm{H}, \mathrm{CH}_{2}\right) ; 3.23-3.18\left(\mathrm{~m}, 2 \mathrm{H}, \mathrm{CH}_{2}\right)$; 2.01-1.93 (m, 2H, CH$) ; 1.84-1.75\left(\mathrm{~m}, 6 \mathrm{H}, \mathrm{CH}_{2}\right) .{ }^{13} \mathrm{C}-\mathrm{NMR}\left(\mathrm{CDCl}_{3}\right): \delta=154.8(\mathrm{C} 5 \mathrm{a}) ; 148.3(\mathrm{q}$, $\left.{ }^{2} J_{\mathrm{C}-\mathrm{F}}=31.9, \mathrm{C} 11\right) ; 146.9(\mathrm{C} 4 \mathrm{a}) ; 146.8(\mathrm{C} 2) ; 135.3(\mathrm{C} 10 \mathrm{a}) ; 132.7(\mathrm{C} 3) ; 126.6(\mathrm{C} 11 \mathrm{a}) ; 122.7$ (q, $\left.{ }^{1} J_{\mathrm{C}-\mathrm{F}}=277.0, \mathrm{CF}_{3}\right) ; 122.4(\mathrm{C} 4) ; 120.2(\mathrm{C} 1) ; 31.3\left(\mathrm{CH}_{2}\right) ; 28.4\left(\mathrm{q},{ }^{4} J_{\mathrm{C}-\mathrm{F}}=4.0, \mathrm{C} 10\right) ; 28.3\left(\mathrm{CH}_{2}\right)$; $25.8\left(\mathrm{CH}_{2}\right) ; 25.6\left(\mathrm{CH}_{2}\right)$. CG-MS (EI, $\left.70 \mathrm{eV}\right): \mathrm{m} / \mathrm{z}(\%)=310\left(\mathrm{M}^{+}, 100\right) ; 264(6) ; 241(6) ; 195$ (7); 75 (17); 69 (21). Anal. Calcd. for $\mathrm{C}_{15} \mathrm{H}_{13} \mathrm{~F}_{3} \mathrm{~N}_{2} \mathrm{O}_{2}$ (310.09): C, 58.07; H, 4.22; N, 9.03. Found: C, $57.89 ; \mathrm{H}, 4.19 ; \mathrm{N}, 8.85 \%$.

11-Trifluoromethyl-2-chloro-7,8,9,10-tetrahydro-6H-cyclohepta[b]quinoline (6d). Gray solid, yield 15\%, Mp. 133-135 ${ }^{\circ} \mathrm{C} .{ }^{1} \mathrm{H}-\mathrm{NMR}\left(\mathrm{CDCl}_{3}\right): \delta=8.13-8.09(\mathrm{~m}, 2 \mathrm{H}, \mathrm{Ar}) ; 7.66(\mathrm{dd}, 1 \mathrm{H}, J$ $=2.0, J=2.0, \mathrm{Ar}) ; 3.31-3.12\left(\mathrm{~m}, 4 \mathrm{H}, \mathrm{CH}_{2}\right) ; 1.93-1.65\left(\mathrm{~m}, 6 \mathrm{H}, \mathrm{CH}_{2}\right) .{ }^{13} \mathrm{C}-\mathrm{NMR}\left(\mathrm{CDCl}_{3}\right): \delta=$ 151.3 (C5a); 145.3 (q, $\left.{ }^{2} J_{\mathrm{C}-\mathrm{F}}=31.4, \mathrm{C} 11\right) ; 143.3(\mathrm{C} 4 \mathrm{a}) ; 134.4(\mathrm{C} 2) ; 134.2(\mathrm{C} 3) ; 132.3(\mathrm{C} 4) ; 130.0$ (C10a); 128.1 (C11a); 122.2 (q, $\left.{ }^{1} J_{\mathrm{C}-\mathrm{F}}=276.6, \mathrm{CF}_{3}\right) ; 122.1(\mathrm{C} 1) ; 31.3\left(\mathrm{CH}_{2}\right) ; 30.8\left(\mathrm{CH}_{2}\right) ; 28.3(\mathrm{q}$, $\left.{ }^{4} J_{\mathrm{C}-\mathrm{F}}=2.8, \mathrm{C} 10\right) ; 25.9\left(\mathrm{CH}_{2}\right) ; 25.5\left(\mathrm{CH}_{2}\right)$. CG-MS (EI, $\left.70 \mathrm{eV}\right): \mathrm{m} / \mathrm{z}(\%)=299\left(\mathrm{M}^{+}, 100\right) ; 264$ (18); 230(11); 195 (6); 75 (7); 69 (6). Anal. Calcd. for $\mathrm{C}_{15} \mathrm{H}_{13} \mathrm{ClF}_{3} \mathrm{~N}$ (299.07): C, 60.11; H, 4.37; N, 4.67. Found: C, 59.71; H, 4.34; N, 4.58\%.

2-Bromo-11-trifluoromethyl-7,8,9,10-tetrahydro-6H-cyclohepta[b]quinoline (6e). White solid, yield 20\%, Mp. 138-140 ${ }^{\circ} \mathrm{C} .{ }^{1} \mathrm{H}-\mathrm{NMR}\left(\mathrm{CDCl}_{3}\right): \delta=8.28(\mathrm{~d}, 1 \mathrm{H}, J=1.8, \mathrm{Ar}) ; 8.03(\mathrm{~d}, 1 \mathrm{H}$, $J=9.0, \mathrm{Ar}) ; 7.79(\mathrm{dd}, 1 \mathrm{H}, J=2.0 ; J=1.8, \mathrm{Ar}) ; 3.31-3.25\left(\mathrm{~m}, 2 \mathrm{H}, \mathrm{CH}_{2}\right) ; 3.17-3.12(\mathrm{~m}, 2 \mathrm{H}$, $\left.\mathrm{CH}_{2}\right)$; $1.93-1.88\left(\mathrm{~m}, 2 \mathrm{H}, \mathrm{CH}_{2}\right) ; 1.75-1.70\left(\mathrm{~m}, 4 \mathrm{H}, \mathrm{CH}_{2}\right) .{ }^{13} \mathrm{C}-\mathrm{NMR}\left(\mathrm{CDCl}_{3}\right): \delta=151.2(\mathrm{C} 5 \mathrm{a})$; $145.5\left(\mathrm{q},{ }^{2} J_{\mathrm{C}-\mathrm{F}}=31.4, \mathrm{C} 11\right) ; 143.6(\mathrm{C} 4 \mathrm{a}) ; 134.2(\mathrm{C} 3) ; 132.6(\mathrm{C} 4) ; 132.4(\mathrm{C} 10 \mathrm{a}) ; 128.6(\mathrm{C} 11 \mathrm{a})$; $122.2\left(\mathrm{q},{ }^{1} J_{\mathrm{C}-\mathrm{F}}=276.6, \mathrm{CF}_{3}\right) ; 125.5(\mathrm{C} 1) ; 122.8(\mathrm{C} 2) ; 31.3\left(\mathrm{CH}_{2}\right) ; 28.3\left(\mathrm{q},{ }^{4} J_{\mathrm{C}-\mathrm{F}}=3.0, \mathrm{C} 10\right) ; 27.8$ $\left(\mathrm{CH}_{2}\right) ; 26.0\left(\mathrm{CH}_{2}\right) ; 25.6\left(\mathrm{CH}_{2}\right)$.

CG-MS (EI, $70 \mathrm{eV}): m / z(\%)=343\left(\mathrm{M}^{+}, 100\right) ; 274$ (8); 264 (14); 202 (28); 195 (13); 152 (22); 75 (12); 69 (12). Anal. Calcd. for $\mathrm{C}_{15} \mathrm{H}_{13} \mathrm{BrF}_{3} \mathrm{~N}$ (343.02): C, 52.35; H, 3.81; N, 4.07. Found: C, $52.40 ; \mathrm{H}, 3.79 ; \mathrm{N}, 3.97 \%$.

\section{Acknowledgements}

The authors thank Conselho Nacional de Desenvolvimento Científico e Tecnológico - CNPq for financial support (Proc. nr. 473.864/2006-1). A fellowship from the Coordenação de Aperfeiçoamento de Pessoal de Nível Superior - CAPES (T.S.M.) is also acknowledged.

\section{References}

1. Muscia, G. C.; Bollini, M.; Carnevale J. P.; Bruno A. M.; Asís, S. E. Tetrahedron Lett. 2006, 47, 8811 .

2. (a) Gringauz, A. Introduction to Medicinal Chemistry. How Drugs Act and Why, Wiley: Chichester, 1997. (b) Gobeil, R. J.; Hamilton, C. S. J. Am. Chem. Soc. 1945, 67, 511. 
3. De, S. K. ; Gibbs, R. A. Tetrahedron Lett. 2005, 46, 1647.

4. Banwell, M. G.; Lupton, D.W.; Ma, X.; Renner, J.; Sydnes, M. O. Org. Lett. 2004, 6, 2741.

5. Filler, R. In Banks, R. E., Ed. Organofluorine Chemicals and Their Industrial Applications, Ellis Horwood: London, 1979.

6. Inouye, Y.; Tezuka, K.; Takeda, W.; Sugai, S. J. Fluorine Chem. 1987, 35, 275.

7. Cunico, W.; Cechinel, C. A.; Bonacorso, H. G.; Martins, M. A. P.; Zanatta, N.; Souza, M. V. N. de.; Freitas, I. O.; Soares, R. P. P.; Krettli, A. U. Biorg. Med. Chem. Lett. 2006, 16,649 .

8. Cho, C. S.; Kim, B. T.; Kim, Tae-Jeong; Shim, S. C. Chem. Commun. 2001, 2576.

9. Bonacorso, H. G.; Duarte, S. H. G.; Zanatta, N.; Martins, M. A. P. Synthesis 2002, 1037.

10. Bonacorso, H. G.; Drekener, R. L.; Rodríguez, I. R.; Vezzosi, R. P.; Costa, M. B.; Martins, M. A. P., Zanatta, N. J. Fluorine Chem. 2005, 126, 1384.

11. Bonacorso, H. G.; Costa, M. B.; Moura, S.; Pizzuti, L.; Martins, M. A. P.; Zanatta, N.; Flores, A. F. C. J. Fluorine Chem. 2005, 126, 1396.

12. Hojo, M. Masuda, R.; Okada, E. Synthesis 1986, 1013.

13. Hojo, M.; Masuda, R.; Kokuryo, Y.; Shioda, H.; Matsuo, S. Chem. Lett. 1976, 499.

14. Flores, A. F. C.; Siqueira, G. M.; Freitag, A. R.; Zanatta, N.; Martins, M. A. P. Quim. Nova 1994, 17, 298.

15. Poszavacz, L.; Simig, G. Tetrahedron 2001, 57, 8573.

16. Tyvorsky, V. I.; Bodrov, D. N.; Kulinkovich, O. G.; Aelterman, W.; Kimpe, N. D. Tetrahedron 2000, 56, 7313.

17. Hojo, M.; Masuda, R.; Tomifuji, T.; Imazaki, N. Synthesis 1990, 1135. 